\title{
Obstructive sleep apnoea: A review of the orofacial implications
}

\author{
David Sherring, ${ }^{*}$ Norman Vowles, $†$ Ral Antic, ${ }^{*}$ * Suren Krishnan, $\mathbb{I l}$ Alastair N Goss $<$
}

\section{Abstract}

Obstructive sleep apnoea is a complex multifactorial condition produced by a combination of anatomical and physiological factors. There is a significant associated mortality and morbidity to obstructive sleep apnoea.

There is an at least 25 per cent increased mortality from cardiovascular disease when obstructive sleep apnoea patients are compared to age and gender matched healthy people. Obstructive sleep apnoea sufferers also have a much higher industrial and motor vehicle accident rate.

Management of the condition should be undertaken by a multidisciplinary team including respiratory physicians, sleep laboratory technicians, otorhinolaryngologists, oral and maxillofacial surgeons and dental specialists. The diagnostic and therapeutic interactions of team members are the key to successful treatment. The treatment regime utilises nasal continuous positive airway pressure devices, mandibular advancement splints and soft and hard tissue surgery.

This review provides the dental practitioner with an introduction to obstructive sleep apnoea with particular emphasis on the orofacial aspects.

Key words: Obstructive sleep apnoea, mandibular repositioning appliances, orthognathic surgery.

(Received for publication January 2001. Revised May 2001. Accepted May 2001.)

\section{INTRODUCTION}

Sleep is normal, essential and one of the major pleasures of life. Most people at some time in their life have experienced a period of acute sleep deprivation, whether from pain, anxiety, long-distance travel or environmental noise and have first hand experience of the rapid onset of emotional and physical instability.

\footnotetext{
"Registrar, Oral and Maxillofacial Surgery Unit, Adelaide University. †Research Fellow, Oral and Maxillofacial Surgery Unit, Adelaide University.

* Director, Forensic Medicine Unit, Royal Adelaide Hospital. IISenior Specialist, Otorhinolaryngology Unit, Royal Adelaide Hospital.

$<$ Professor and Director, Oral and Maxillofacial Surgery Unit, Adelaide University.
}

Sleep deprivation, however, is but one of many sleep disorders which have been the subject of scientific and clinical study in sleep laboratories worldwide.

One of the common sleep disorders is obstructive sleep apnoea (OSA). Obstructive sleep apnoea results in disturbed sleep, snoring and daytime sleepiness. About 40 per cent of the adult population snore loudly on a regular basis and approximately 4 per cent of men and 2 per cent of women suffer OSA. ${ }^{1}$ Over the last two decades, studies have shown that these apparently annoying and innocuous symptoms are associated with significant morbidity and mortality. This finding has led to increased interest in the aetiology and management of this multifactorial condition.

In some but not all cases of OSA, there is an associated retrusion of the facial skeleton and/or increased soft tissue volume of the oropharyngeal tissues. This condition has led to the development of a range of non-surgical and surgical techniques to reposition and change the shape of these tissues. Initially, good results have been reported with a wide range of techniques but often on a very subjective and anecdotal basis - the longer term results are usually not so good.

The use of oral appliances to treat snoring and OSA is of particular relevance to the dental profession. These appliances are being actively promoted to the profession and some may see these appliances as a means of broadening their treatment scope. The use of oral appliances is the subject of a joint Australian Dental Association and Thoracic Medicine Society of Australia and New Zealand statement and this review provides the more detailed scientific background to this statement. ${ }^{2-3}$

The authors of this review (RA, SK, AG) have worked in the area of sleep disorders for many years. Three of the authors meet regularly at the Royal Adelaide Hospital to plan patient services, assess individual management problems and conduct clinical investigations. Specialist trainees from their respective units are actively involved. One of the authors (DS) has performed an extensive study on craniofacial morphology in OSA patients. The remaining author (NV) is an associate of the sleep disorders unit and has 
Table 1. Sleep stages.

\begin{tabular}{ll}
\hline Awake & $\begin{array}{l}\text { EEG alpha waves }(8-12 \mathrm{~Hz}) \text { eyes open } \\
\text { beta waves }(14+\mathrm{Hz}) \text { eyes closed }\end{array}$ \\
\hline Stage 1 & $\begin{array}{l}\text { Initial, transient phase } \\
\text { theta waves }(3-6 \mathrm{~Hz})\end{array}$ \\
$5 \%$ & Predominant phase of NREM sleep \\
Stage 2 & Complex sleep spindle, \\
$50 \%$ & Waxing and waning $12-14 \mathrm{~Hz}$ \\
Stage 3 & 20-50\% \\
$10 \%$ & High amplitude $(>75 \mathrm{~V})$ \\
& 20\% slow frequency $(2 \mathrm{~Hz})$ \\
Stage 4 & $>50 \%$ high amplitude \\
$10 \%$ & Slow frequency \\
REM sleep & Onsets around 90 minutes $-25 \%$ \\
$25 \%$ & Associated with dreams \\
\hline
\end{tabular}

recently taken over coordination of the oral appliance studies at the oral and maxillofacial surgery unit.

In this paper, the key areas of the literature are reviewed. The key areas are normal sleep; sleep laboratory; OSA, the morbidity and mortality of OSA, upper airway anatomical and physiological considerations in OSA; clinical investigation and treatment, including oral appliances and hard and soft tissue surgery. This review is illustrated by clinical and research experience.

\section{Normal sleep}

The average adult requires six-eight hours' sleep per 24-hour cycle, although the individual requirement is widely variable. It is a complex, physiological process with four repeating stages (Table 1). These stages are characterised by phases of rapid eye movement (REM) sleep and non-rapid eye movement (NREM) sleep with different types of brainwaves as a measure of electrical brain activity (EEG). Generally, there are four to five cycles of NREM/REM sleep during an eight-hour period of sleep. ${ }^{4}$ These cycles of sleep are associated with several concurrent physiological processes. ${ }^{5}$ Muscle tone generally decreases but with short bursts of increased limb activity. These bursts of limb activity result in changes of body position which usually occur prior to and emerging from periods of REM sleep.

The heart rate decreases in NREM sleep but is variable in REM sleep but the overall result is similar to baseline awake levels. In NREM sleep, there are changes in atrial ventricular (AV) conduction and increased refractory periods. Blood pressure decreases by up to 20 per cent of baseline awake levels in normotensive and hypertensive individuals in NREM sleep.

Table 2. Polysomnography.

\begin{tabular}{lc}
\hline Electrooculogram & EOG \\
Electroencephalogram & EEG \\
Electrocardiogram & ECG \\
Electromyogram - submental and tibialis anterior & EMG \\
Oronasal airflow & \\
Pulse oximetry - arterial $\mathrm{O}_{2}$ saturation & \\
Respiratory inductive plethysomograph - chest and & \\
abdominal movement & \\
Microphone - snoring & \\
Video recording - body movement & \\
\hline
\end{tabular}

Australian Dental Journal 2001;46:3.

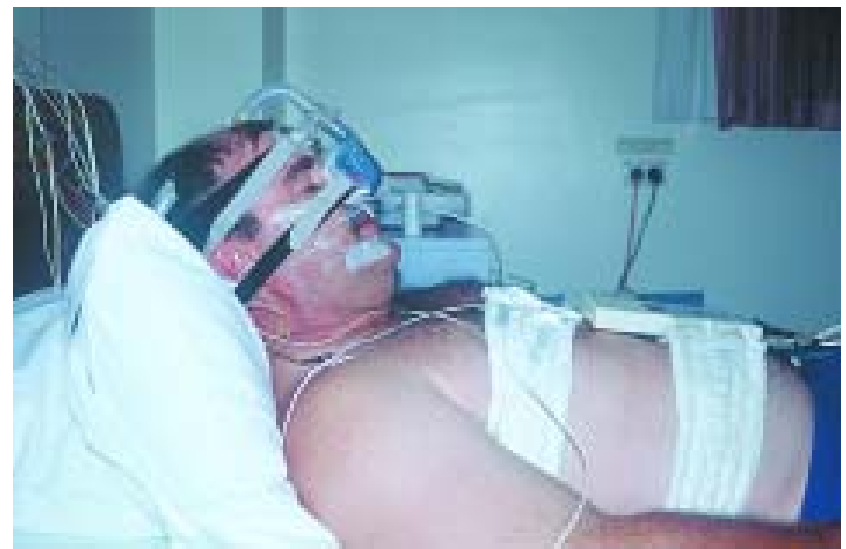

Fig 1. Patient being monitored in a sleep laboratory.

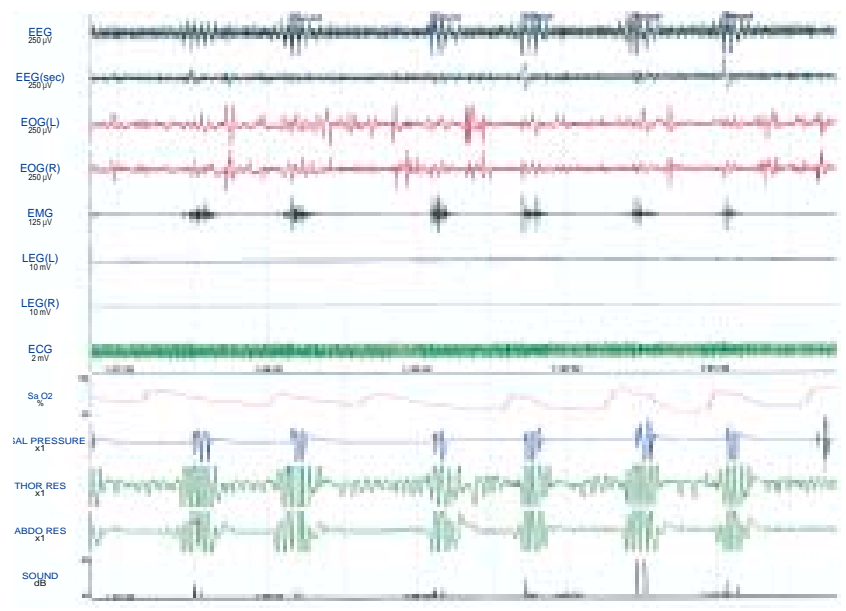

Fig 2. Polysomnogram record. The patient had six arousals in four minutes that followed obstructive events involving no nasal airflow but persisting thoracic and abdominal effort.

In NREM sleep, respiratory drive decreases but airway resistance increases from the decreased muscle activity of the upper airway muscles. In the transition from wakefulness to NREM sleep, airway resistance can increase two-three times. Gaseous exchange in the pulmonary alveoli alters, with the $\mathrm{PCO} 2$ increased by $2-8 \mathrm{~mm}$ mercury and a fall of $3-10 \mathrm{~mm}$ mercury in PO2 compared to baseline awake levels.

The overall metabolic rate decreases by $10-20$ per cent in NREM sleep compared to baseline awake levels. The implication is that sleep allows the body to rest and repair for the next day's activities.

\section{Sleep laboratory}

Sleep laboratories measure the various physiological parameters of sleep. The common parameters are listed in Table 2. Precisely which parameters are measured depends on financial considerations and whether the laboratory is scientifically or clinically orientated.

Essentially, the patient presents in the early evening and has various monitoring devices attached and calibrated. These devices monitor the patient while they are asleep (Fig 1, 2, 3).

The following morning, the patient leaves for work or home. The records are reviewed and reported by the 


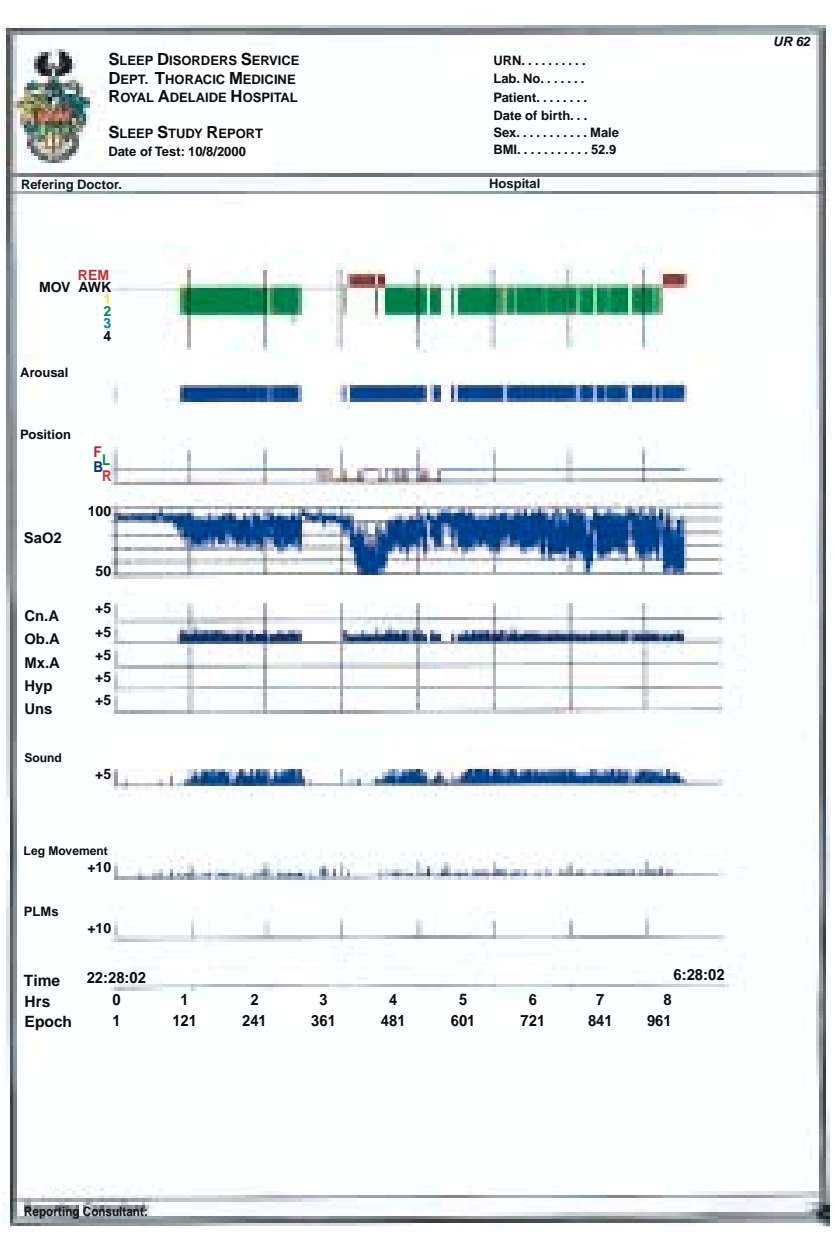

Fig 3. Sleep study report summarising an overnight study.

sleep laboratory technician and by a respiratory physician, trained in the management of sleep disorders and the interpretation of sleep studies.

Sleep studies can be repeated to assess whether treatment has controlled the disordered OSA. Thus, for example, nasal continuous positive airway pressure (nCPAP) can be titrated, mandibular repositioning appliances (MRA) worn in a split study to test its effect or post surgery compared to the initial study.

Patients will sometimes comment that a sleep laboratory is a different environment to sleeping at home. This factor is taken into account in the interpretation of sleep studies. Only very limited investigations can be performed at the patient's home, so home studies are usually not clinically useful.

Table 3. International classification of sleep disorders. These major categories are further subdivided into 84 separate subcategories.

\begin{tabular}{ll}
\hline Dyssomnias & Intrinsic sleep disorders \\
& Extrinsic sleep disorders \\
& Circadian rhythm sleep disorders \\
Parasomnias & Arousal disorders \\
& Sleep-wake transition disorders \\
& Parasomnias usually associated with \\
& REM sleep \\
& Other parasomnias \\
Medical/psychiatric & Associated with mental, neurologic \\
sleep disorders & and other mental disorders \\
\hline
\end{tabular}

\section{Obstructive sleep apnoea}

Sleep apnoeas are dyssomnias ${ }^{6}$ (Table 3 ) and can be subdivided into three groups: central, obstructive or mixed central and obstructive. Central apnoeas are neurological in origin and won't be discussed in this review.

In $1990,{ }^{6}$ OSA was defined by the American Sleep Disorders Association as being 'characterised by repetitive episodes of upper airway obstruction that occur during sleep, usually associated with a reduction in oxygen saturation, daytime sleepiness and snoring'. This is a very broad definition which requires further characterisation. The obstructive episodes are of two types, apnoea and hypopnoea. Apnoeas are episodes of total airway obstruction which last for more than 10 seconds. Hypopnoeas are partial airway obstructions.

A variant and probably part of the spectrum of disordered breathing from uncomplicated snoring to OSA is upper airway resistance syndrome. This syndrome is found in extremely sleepy patients with a short time to sleep onset and an excess of arousals. Increased respiratory efforts and inspiratory flow limitations are indicative of the syndrome but with no excess of hypopnoeas or apnoeas. Clinically, the management of hypopnoeas or apnoeas is the same as the management of OSA.

The number of apnoeic episodes per hour is called the apnoea index (AI). ${ }^{7}$ Hypopnoeas are characterised by partial obstruction and are quantified by using the respiratory disturbance index (RDI) or apnoea/ hypopnoea index (AHI), ${ }^{8}$ that is, the number of apnoeas and hypopnoeas per hour in sleep.

The staging of OSA is not fully agreed in the literature. The presence of an $\mathrm{AI}>5$ with daytime somnolence is regarded by some as OSA and treatment is offered to those with an RDI of $>15$ with symptoms of OSA and comorbid conditions such as cardiovascular disease. All patients with an RDI $>30$ are offered treatment.

The principal cardiovascular effects of OSA which occur during apnoeas are reduction in cardiac output, dysrhythmias and nocturnal hypertension. ${ }^{9}$ The respiratory effects are a decrease in oxyhaemoglobin saturation, faster desaturation and ventilation perfusion mismatching with decreased lung oxygen stores.

The morbidity and mortality of OSA

Untreated OSA has an increased mortality with a number of studies showing a 25-37 per cent increased mortality rate over an eight-year period. ${ }^{10-11}$ These deaths are most commonly attributed to myocardial infarction or cerebrovascular accidents. Death may also be associated with pulmonary oedema, pulmonary hypertension and obstructive airway disease.

Hypersomnolence or daytime sleepiness is also a significant factor in acute death from OSA. The act of falling asleep while driving or operating machinery has been associated with OSA. ${ }^{12-13}$ Using driver simulation, OSA subjects ran off the road 101 times compared to 
Table 4. Morphologic state associated with OSA.

Maxillary retrusion
Mandibular retrusion
Tonsillar and adenoid hypertrophy
Glottic web
Vocal cord paralysis
Acromegaly
Lymphoma
Ectopic thyroid
Severe kyphoscoliosis
Cushings syndrome
Stroke

twice for non-OSA subjects. ${ }^{14}$ Another study reported 71 per cent of OSA patients having had at least one motor vehicle accident of which 13 per cent were directly attributed to falling asleep. The OSA group were also more likely to near-miss, with 69 per cent indicating an average of nine near-accidents while nonOSA controls averaged only one near-accident..$^{15}$ As OSA sufferers could fall asleep while driving or working machinery, they pose a significant risk to other members of the community.

Obstructive sleep apnoea neurological and endocrine complications include elevated fasting insulin levels, impaired spatial orientation and recall. ${ }^{16-17}$

Obesity and OSA are inextricably linked. ${ }^{18}$ Obese patients have increased cardiovascular mortality and difficulty in exercising, leading to an increased sedentary lifestyle with further increases in weight. Fat also becomes deposited in the soft palate and oropharynx and increases the degree of obstruction. However, 10 per cent weight loss may result in a 50 per cent improvement in respiration. ${ }^{19}$

Upper airway anatomical and physiological considerations in OSA

Any anatomical or pathological change which reduces the size of the upper airway may result in obstruction of the airway and thus predispose to OSA (Table 4).

Any physiological change that reduces the ability of the pharyngeal muscles to maintain an open airway may predispose to OSA. Some of the physiological and pathological states identified as being associated with OSA are listed in Table 5.

There have been numerous research investigations into the anatomical and physiological status of the hard and soft tissues of the face, jaws and upper airway. These investigations include direct examination, ${ }^{20}$ endoscopy ${ }^{21}$ fluoroscopy, cephalometry, CT scans and magnetic resonance imaging. ${ }^{22}$ All these investigative

\section{Table 5. Functional states associated with OSA.}

\section{Encephalitis \\ Epilepsy \\ Motor neurone diseases \\ Dysautonomia \\ Alveolar hypoventilation \\ Hypothyroidism \\ Testosteronism \\ Sedative/hypnotic agents}

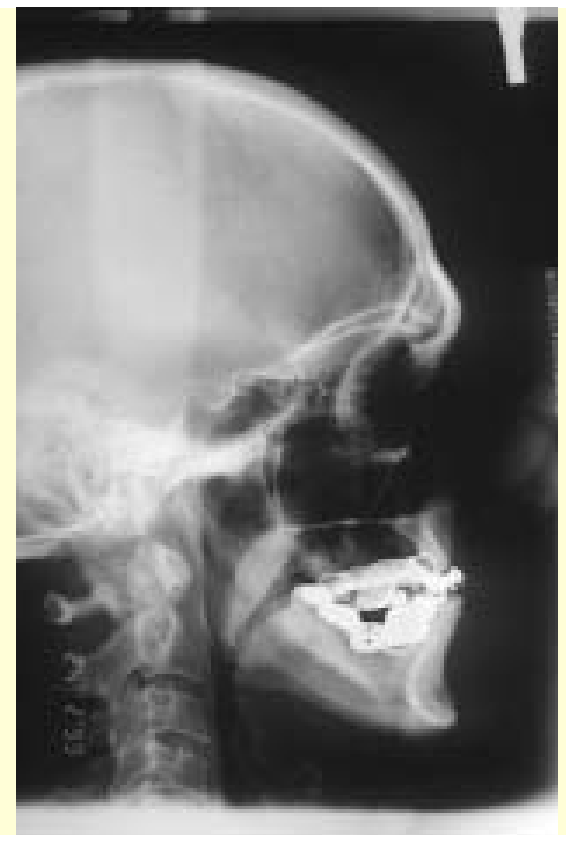

Fig 4. Lateral head cephalogram. Note the retruded maxilla and long, fat soft palate.

methods have their advantages and disadvantages and most have contributed to the sum of knowledge of the condition. The most common difficulty faced by researchers is whether a conscious investigation shows what is actually happening when the patient is asleep. This is particularly the case in relation to pathological changes to the physiology rather than anatomical changes. Other unresolved issues include if the population studied is representative and comparable to other studies.

In a recent trial, 94 patients who clinically snored and had a full sleep study workup underwent lateral head cephalometric examination. ${ }^{23}$ The patients were subdivided into those who snore and had OSA (59 patients) and those snorers who did not suffer OSA (35 patients). Demographically, the two groups were similar. The lateral head cephalogram (Fig 4) was analysed in detail for the hard and soft tissue (Fig 5, 6) dimensions and these were correlated to the sleep study. The main findings, when OSA patients were compared to non-OSA snorers, were an increased incidence of maxillary retrusion $(\mathrm{ANB}<0)$; an increased incidence of mandibular retrusion $(\mathrm{ANB}>0)$; an increased incidence of maxillary and mandibular retrusion (SNA and SNB); the hyoid was more inferiorly and anteriorly placed; a thicker soft palate; a larger tongue; a longer pharyngeal length; and there was no significant difference in the posterior airway space (PAS) between the two groups.

The craniofacial morphology differences between snorers and OSA patients are presented in Table 6. There were also correlations between the basal metabolic rate and craniofacial morphology. Generally, obese patients showed less anatomical anomalies than normal weight patients who had more retruded facial skeletons. The most severe OSA was found in obese patients with concurrent facial retrusion. 


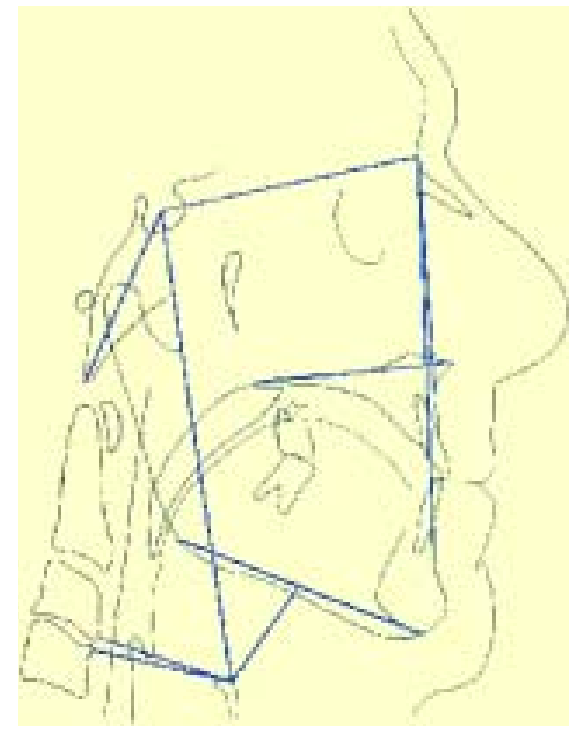

Fig 5. Hard tissue landmarks. Cranial base, maxilla and mandible length and AP position, hyoid position.

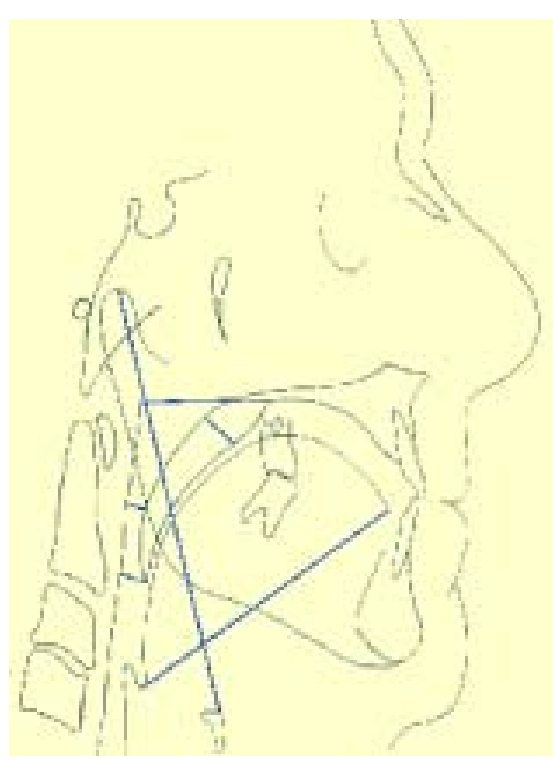

Fig 6. Soft tissue landmarks. Soft palate, upper airway, tongue and oesophagus.
Table 6. Craniofacial morphology difference between snorers (RDI <10) and OSA (RDI >10).

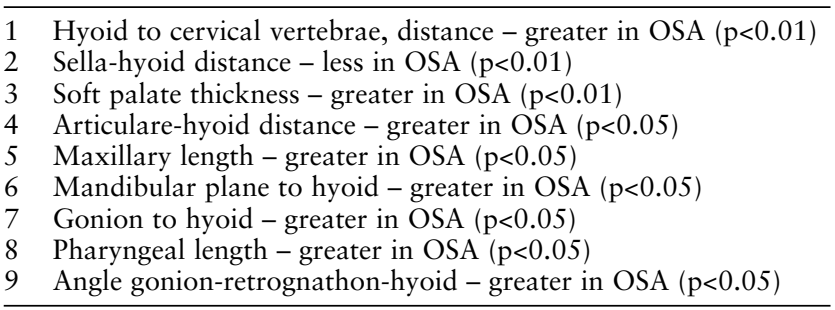

\section{Clinical investigation and treatment}

The key step in the management of OSA is to firmly establish the diagnosis. Questionnaires may, however, give an initial measure of the nature of the problem (Table 7). Definitive diagnosis requires specialist thoracic medicine investigation and in most cases a sleep study (Table 8). There is a risk of underdiagnosing the patient for whom the presenting complaint is snoring as severe snorers and patients with obstructive sleep apnoea often appear similar. However, snoring is a disturbance for the spouse and/or neighbours whereas OSA has a significantly increased risk of morbidity and mortality.

Otorhinolaryngologists (ORL) have an important role in the diagnosis and management of snoring and OSA. Indeed, they may commonly be the first specialist consulted. The ORL's contribution is to determine the anatomy, function and pathology of the upper airway from the nose to larynx. Investigations should include a full history and examination, imaging and direct examination of the airway. There is a wide range of developmental, traumatic, inflammatory and neoplastic pathology which may present as upper airway obstruction (some are illustrated in Fig 7).

It should be noted that some patients have been diagnosed and treated as snorers when, in fact, they had cancers of the nose or pharynx. Therefore, full airway examination should be mandatory.

Fibreoptic nasendoscopy allows complete inspection of the upper airway. Muller's manoeuvre, where the patient inspires with the mouth and nose closed, allows inspection of the site of collapse at the tongue base or velopharyngeal valve. More usefully, sleep endoscopy, where the patient is given intravenous sedation to

Table 7. The Epworth score - a measure of daytime sleepiness. A score of 10 or more indicates there may be a problem and formal investigation is indicated. How do you score?

\begin{tabular}{lccc}
\hline Situation & Would never doze & Slight chance of dozing & Mod chance of dozing High chance of dozing \\
Score 0 & Score 1 & Score 2 & Score 3
\end{tabular}

Sitting and reading

Watching TV

Sitting, inactive, in a public place

(for example, a theatre or a meeting)

As a passenger in a car for an hour

without a break

Lying down to rest in the afternoon

when circumstances permit

Sitting and talking to someone

Sitting quietly after a lunch without alcohol

In a car, while stopped for

a few minutes in traffic 
Table 8. Medical investigation.

\section{Presenting complaint}

Initial questionnaire (Epworth score)

Medical history

General medical examination

Head and neck examination

Cephalometry

Nasendoscopy

Polysomnography

Diagnostic nCPAP

induce snoring, allows direct visualisation of the site of obstruction. ${ }^{24}$ Patients may have a single or multiple site of obstruction, therefore sleep nasendoscopy is a useful adjunct in determining the proper course of treatment.

The dental specialist's role is to determine the patient's facial bone morphology both clinically and cephalometrically. The hard and soft tissue relationships need to be analysed carefully as soft tissue surgery in the presence of untreated skeletal problems is inappropriate. The temporomandibular joint and muscle function also need to be assessed.

The essential function of the various specialist examinations is not only to determine problems in one's own field of expertise but also to communicate findings with other members of the team. Hence, if a patient presents to the dentist for treatment of snoring, whether they be self-referred, general medically referred or referred by a sleep laboratory, the dentist must be fully cognisant of the nature of the problem and the
Table 9. Indications for treatment.

\begin{tabular}{l} 
RDI $>20$ \\
Oxygen desaturation $<90 \%$ \\
Comorbidities \\
$\quad$ - hypertension \\
- heart disease \\
- arrhythmias \\
\hline
\end{tabular}

workup required. If this preparatory work has not been done, then it should be arranged before initiation of any treatment. ${ }^{2}$

A wide range of treatments has been reported as being of some benefit for OSA patients. The general indications for treatment of OSA are listed in Table 9 and the criteria for successful treatment in Table 10. The simplest treatment regimes involve changes in sleep position from the supine to lateral cubitus positioning which can result in a halving of the AHI. ${ }^{25}$ This change in sleep position is worth a try for normal-weight snorers although it has usually been tried before a patient presents to a sleep disorders unit. The cessation of respiratory depressant medications and decreasing alcohol intake are important aids in combating OSA. In obese patients, weight loss is an obvious goal but often hard to achieve on a long-term basis. ${ }^{18-19}$

The gold standard treatment for OSA is nCPAP (Fig 8). ${ }^{26}$ Essentially, nCPAP treatment utilises an aircompressor unit that directs airflow down the patient's
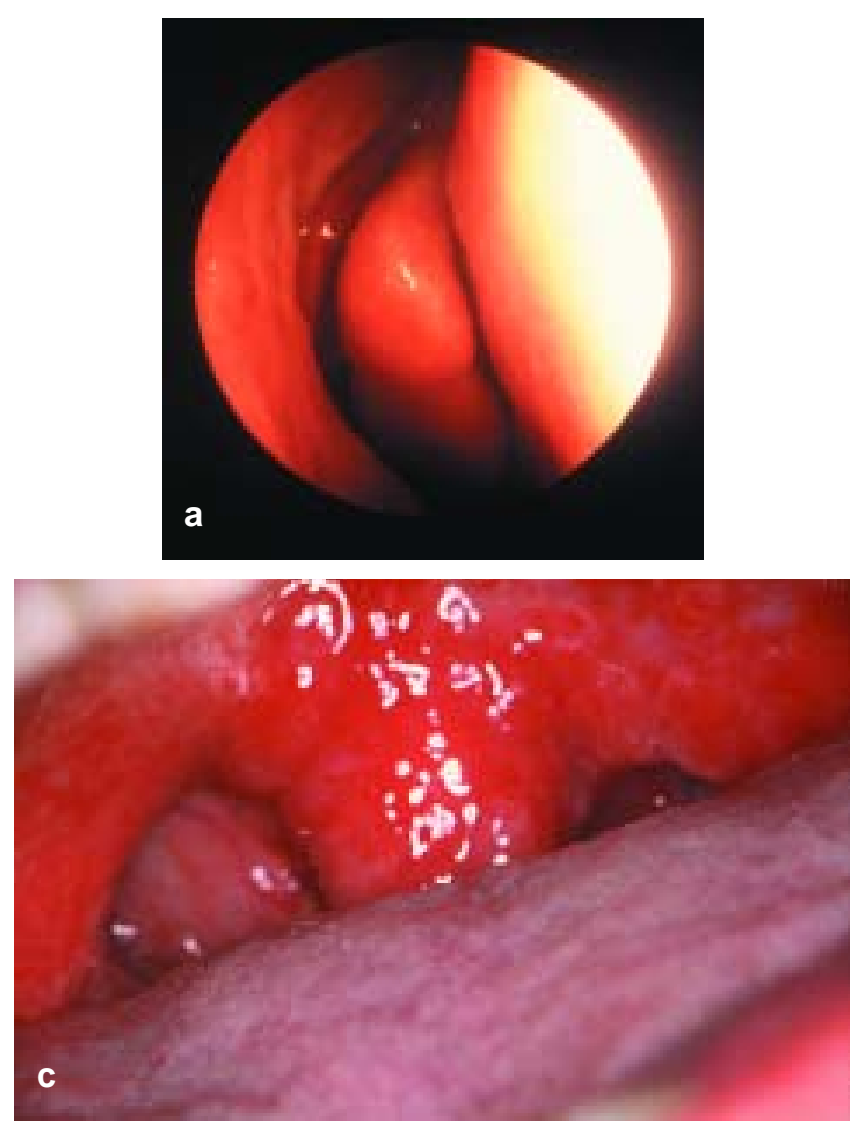

Fig 7. Upper airway conditions which may give rise to the signs and symptoms of snoring or OSA: (a) deviated nasal septum and concha bullosa (enlarged turbinate); (b) nasal polyp; (c) enlarged right tonsil and uvula; and (d) large, right-sided, deep lobe parotid tumour (CT scan).
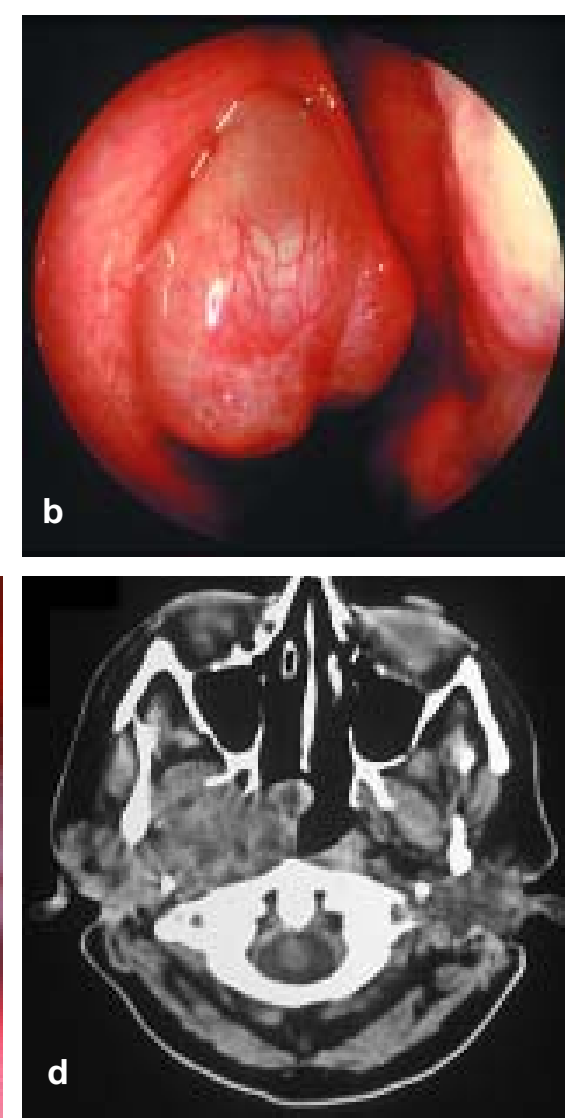

d 
Table 10. Criteria for successful treatment.

\section{Results equal to nCPAP}

RDI less than 10

Oxygen desaturation above $90 \%$

No excessive daytime sleepiness

airway via the nose, with the mouth closed. The increased air pressure holds the airway open. The degree of positive pressure needed is titrated during a sleep study. Regular nCPAP treatment for at least five hours a night has been shown to control hypersomnolence. During treatment, snoring is contained and AI and RDI significantly improved. This treatment has been shown to reduce driving problems and should, in the long term, reduce the cardiovascular mortality and morbidity of OSA.

Compliance with the device is the main drawback of nCPAP. There are multiple reasons for discontinuance of treatment, including lack of commitment, resentment of spouse pressure, self-image, noise of the compressor, discomfort of the mask or cost of purchasing the unit. The presence of unrecognised comorbidities such as insomnia, depression, parasomnia or lifestyle issues may lead to lack of control of the presenting symptoms, particularly daytime sleepiness. The acceptance rate of nCPAP has been variously estimated at a low of 46 per cent ${ }^{27}$ to a high of over 90 per cent..$^{28}$ Certainly most patients who are referred for MRA are those who have abandoned nCPAP and thus most ORL and dental team members have a jaundiced view of nCPAP.

\section{Oral appliances}

A variety of appliances have been developed to increase the oropharyngeal airway space. They have included devices with extensions to the soft palate or to the base of the tongue, tongue repositioning devices or, more commonly, MRA.

Cartwright ${ }^{29}$ reported a significant reduction in AHI with a tongue repositioning device. The tongue is secured anteriorly by means of negative pressure from a soft plastic bulb between the lips and teeth but the device is bulky and causes considerable mandibular opening.

The principle behind MRA is that the mandible is held forward during sleep. In so doing, by holding the tongue and pharyngeal muscles forward, the posterior airway space is increased. ${ }^{23}$ Mandibular repositioning appliances have the advantage of being simple, reversible and cost-effective..$^{30}$ There are approximately 50 such appliances on the market.

In an OSA review conducted by the American Sleep Disorders Association, 21 case studies were presented..$^{31-32}$ These studies showed that snoring decreased in nearly all subjects and was often eliminated. The symptoms of OSA were improved in the majority of cases. Approximately 50 per cent of the patients achieved an AHI of less than 20 but, of the remainder, 40 per cent had high and unchanged AHI.

At the Royal Adelaide Hospital, the standard device used for public patients is a monobloc. The monobloc is designed to open the mandible by $5 \mathrm{~mm}$ and to advance the mandible by $7 \mathrm{~mm}$ or three-quarters of maximum protrusion. Generally, 40 per cent of the patients do well, with reasonable long-term compliance, and meet the criteria for successful treatment. A further 40 per cent of patients gain some benefit but either do not like wearing the device or have an insufficiently changed AHI. Approximately 20 per cent of patients abandon the device before its effectiveness is measured.

A series of clinical trials on various oral appliances is

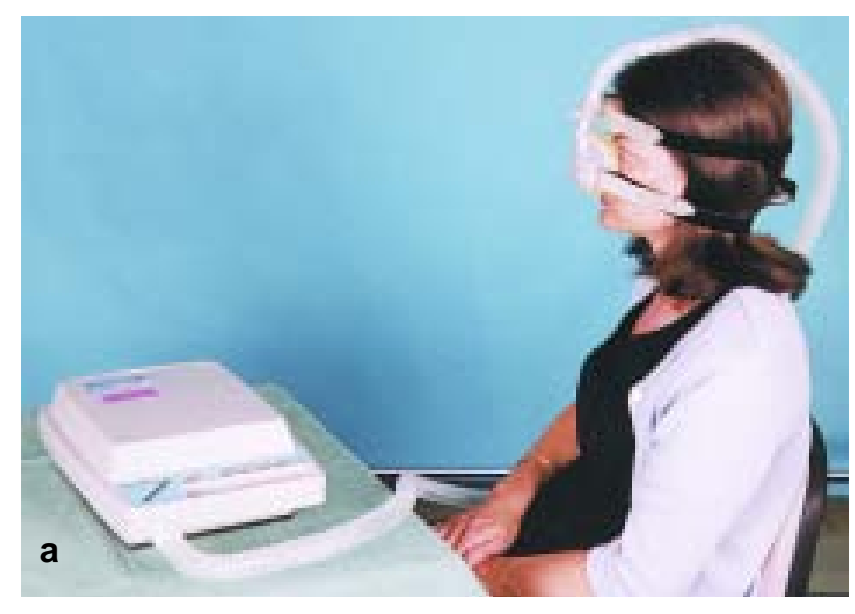

Fig 8. (a) Patient wearing an nCPAP machine, normally they would be wearing this in bed; and (b) closeup of the unit and mask.

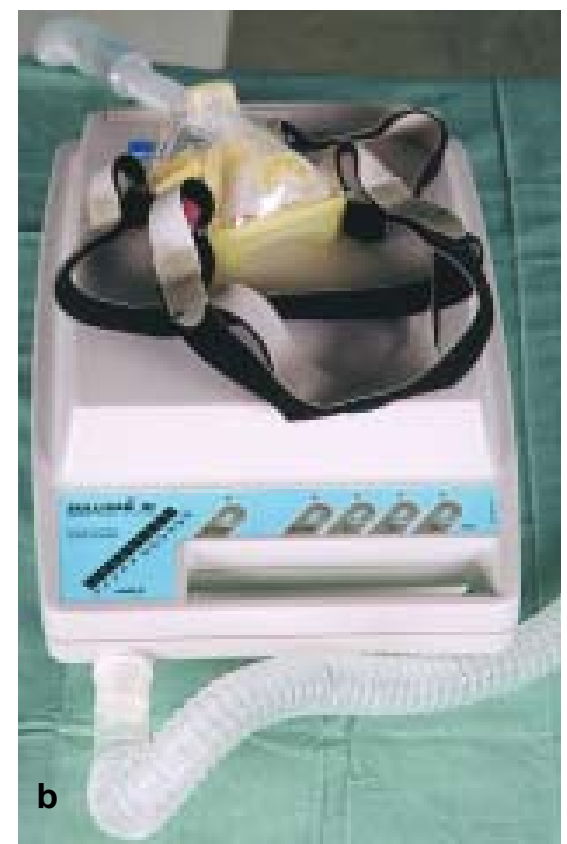



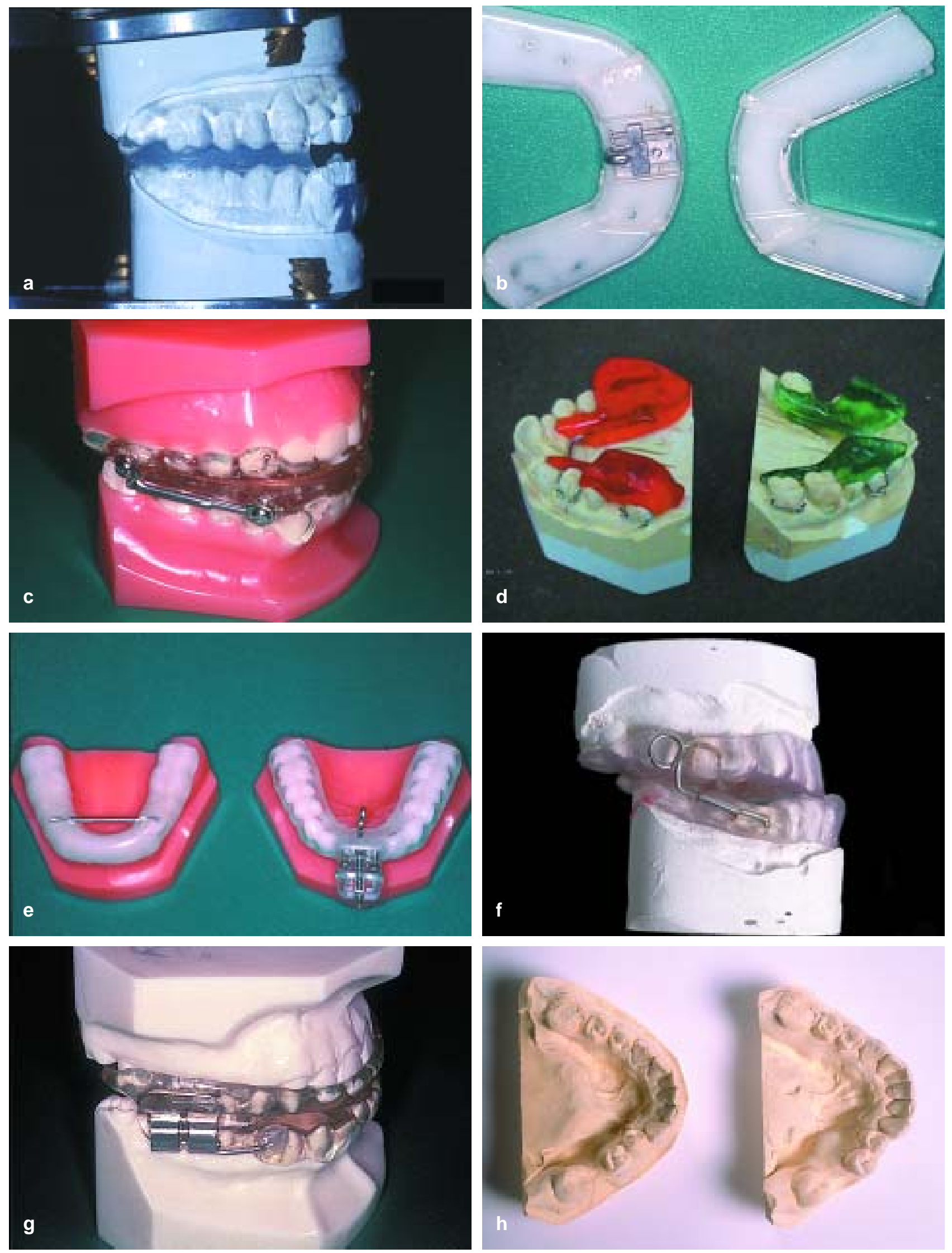

Fig 9. Mandibular repositioning devices: (a) standard monobloc made from mouthguard material; (b) Quiet Knight MRA preformed trays for direct fit on to the teeth, hook and screw adjustment; (c) Herbst appliance, acrylic splint with adjustable metal connectors; (d) twin block acrylic splint with crib retention and ramp anteriorisation; (e) Silent Night, vacuum or heat-formed plastic base, connector of various lengths are used; (f) TAP appliance, dual laminate with adjustable hook and screw attachment; (g) McDonald pattern, dual laminate with adjustable stainless steel rod and tube attachment; (h) study models before (left) and after (right) MRA treatment. If the teeth are not securely held by the splint, uncontrolled orthodontic movement may occur. The right, first premolar was lingually inclined and out of the arch. 

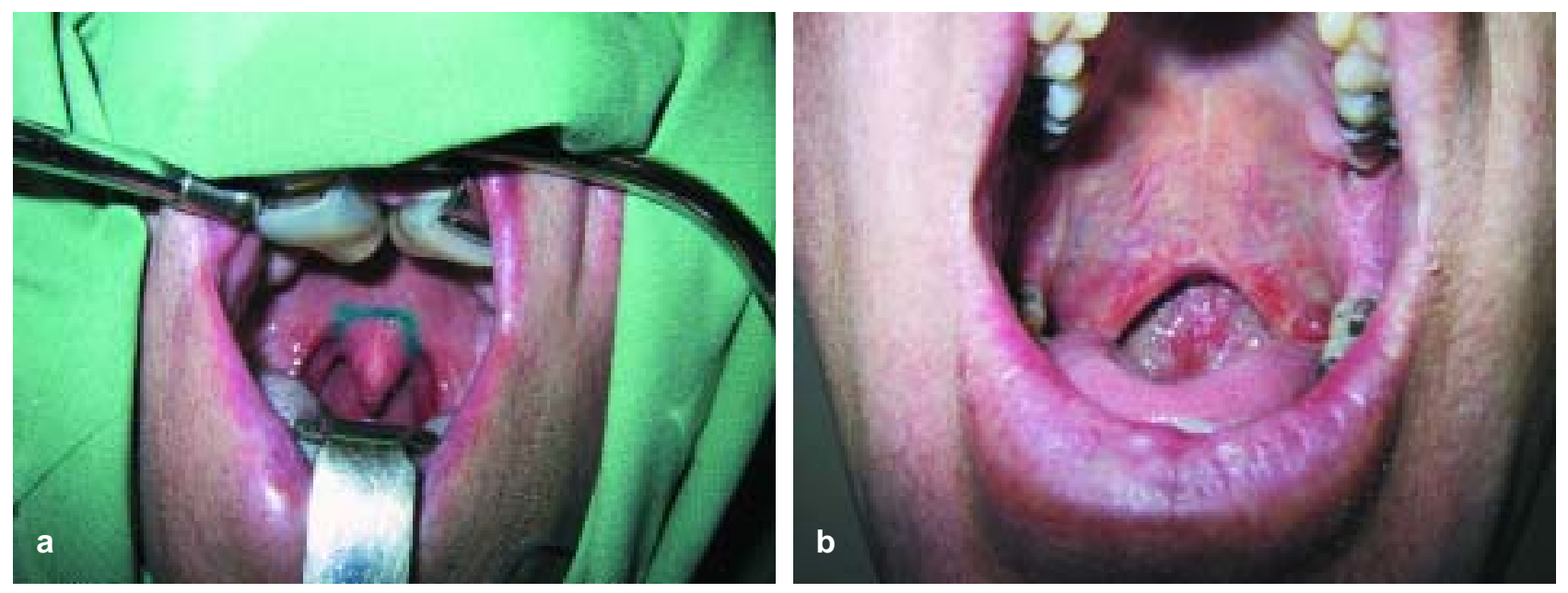

Fig 10. Uvulopalatopharyngoplasty: (a) Intra-operative; and (b) post-operative.

being conducted by one of the authors (NV). In a preliminary study of an adjustable device with a standard tray, which fitted all patients, it was found the results were not as good as the monobloc. The negative results were attributed to the poorly fitting, uncomfortable tray. ${ }^{33}$ The use of customised trays with the same adjustable device has resulted in greater patient acceptance.

It is clear that MRA has a place in the management of OSA. The effects of MRA on the oropharyngeal airway have been well demonstrated by cephalometric radiography and fluoroscopy and it has been shown that while in most cases horizontal mandibular advancement increases the airway space, then jaw opening results in synchronous posterior movement of both the tongue and soft palate. This action results in narrowing of the oropharyngeal airspace. It must be noted that there is a wide variation of the amount of mandibular advancement required to achieve a suitably patent airway. ${ }^{34-36}$

Thus, to be successful, an MRA should be adjustable, produce minimal jaw opening, well fitting, comfortable and robust. The treating dentist must be aware of the medical and social parameters which apply to patients and customise treatment to meet their requirements.

No single device has been demonstrated to be superior to others. Some commonly used devices which meet the design criteria are illustrated in Fig 9.

A range of minor side effects has been reported including temporomandibular disorders (TMD) symptoms, excessive salivation, dry mouth, bruxism, tooth movement and gingival irritation. ${ }^{37-38}$ Generally, all these side effects are mild compared to the complications of obstructive sleep apnoea and can be reversed by adjustment or discontinuing the device.

\section{Hard and soft tissue surgery}

A wide range of surgical options has been described in the ORL and oral and maxillofacial surgery literature. The key point in the surgical management of OSA patients is to be aware that they are very poor anaesthetic and surgical risks. Preoperatively, these patients require full anaesthetic evaluation and, postoperatively, must be admitted for monitoring in an intensive care facility. Due to the risk of morbidity and mortality, sleep endoscopy should not be performed as an office procedure. Even when full precautions are taken, there is still a significant risk of morbidity and mortality. The important dental implication is not to underestimate the risks involved in performing a dentoalveolar procedure on a middle-aged, overweight, snoring patient. The minor office procedure under RA or sedation in an OSA patient may readily convert into a cardiac and/or respiratory arrest.

Tracheostomy is the gold standard surgical method for OSA, as it completely bypasses the upper airway. Tracheostomies do, however, render patients essentially voiceless and have significant complication rates. Tracheostomy is only indicated as the last stage procedure for severe OSA patients.

Obvious pathology of the nasal airway, such as grossly deviated septum, polyps and large tonsils, should be surgically corrected. Uvulo-palatopharyngoplasty (UPPP) (Fig 10) aims to remove the redundant palatal mucosa and widen the pharyngeal fauces. ${ }^{39}$ The initial reports of this technique were promising but subsequent studies, particularly where patients were not carefully selected, showed a success rate of around 50 per cent. ${ }^{40-41}$ The UPPP procedure has significant complications, including palatal incompetence with nasal reflux, altered voice quality, nasopharyngeal stenosis and occasional perioperative mortality relating to haemorrhage and airway obstruction. One further problem is that once a UPPP is performed, it is difficult to reverse the soft tissue changes.

Tongue reduction has been advocated for macroglossia, although there are no good studies on the outcome of this procedure. Hyoid suspension has also been advocated to advance the tongue base where there is no obvious skeletal deficiency. Hyoid suspension is performed by placing a thick, non-resorbable suture or fascia lata graft from the mandible to the tongue. ${ }^{40-41}$ 

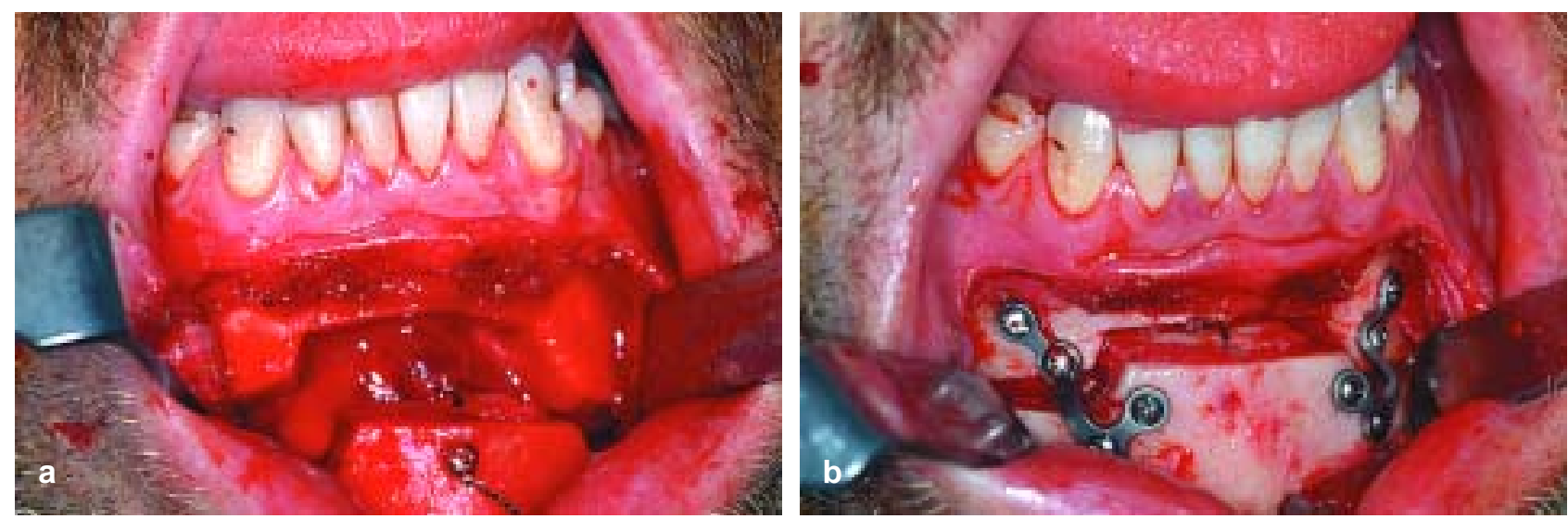

Fig 11. Advancement genioplasty: (a) segment mobilised and brought forward. The midline bone box contains the genial tubercles with the attached pair of genioglossus muscles. The anterior belly of the digastric muscle is attached to the lower border of the advanced chin; and (b) internal fixation of the genial advancement. The osteotomy cuts and screws are at least $4 \mathrm{~mm}$ from the tooth roots.

Hyoid suspension has usually been done in combination with other procedures such as UPPP and/or chin advancement, so there are no good studies on the long-term effect of hyoid suspension.

Palatal stiffening procedures using radiofrequency ablation of the submucosal tissues have been reported as being successful in the reduction of snoring. ${ }^{42}$

Some of the maxillofacial procedures have been reviewed in the Australian literature. ${ }^{43}$ One of these, genial advancement, is a hard tissue operation where a bony window is cut in the chin to isolate the genial tubercles. The bone block with the attached tongue is pulled through and forward to advance the tongue, with a reported success rate of 67 per cent. ${ }^{44-45}$ This operation looks good in diagrams but is technically difficult to perform.

A modification of a standard sliding genioplasty works well (Fig 11). Essentially, the genioplasty is extended upward beneath the incisors to include the genial tubercles. Thus, by this means, the tongue and the anterior digastric muscles are advanced. During the process, the chin segment is secured in place with rigid fixation. This technique is advantageous in that if the position is inappropriate, it can be repositioned.

Genioplasty advancement is only recommended for patients who have had an MRA which has been shown to be effective by a sleep study. A preliminary report showed that subjectively the patients felt better although only 25 per cent had their RDI reduced below $20 .{ }^{46}$

Advancing the mandible alone would usually require pre-surgical orthodontics to correct the occlusion. Hence, mandibular advancement alone is not usually done but is done in combination with a bimaxillary osteotomy. The maxilla is advanced $0.5-1 \mathrm{~cm}$ by a Le Fort I and the mandible then advanced to match the new position of the maxilla. This process usually involves an advancement of the mandible of at least $1 \mathrm{~cm}$ (Figs 12, 13). The literature reports a high $(90+$ per cent) success rate with maxillomandibular advancement. ${ }^{47-49}$ In the preliminary report, high subjective
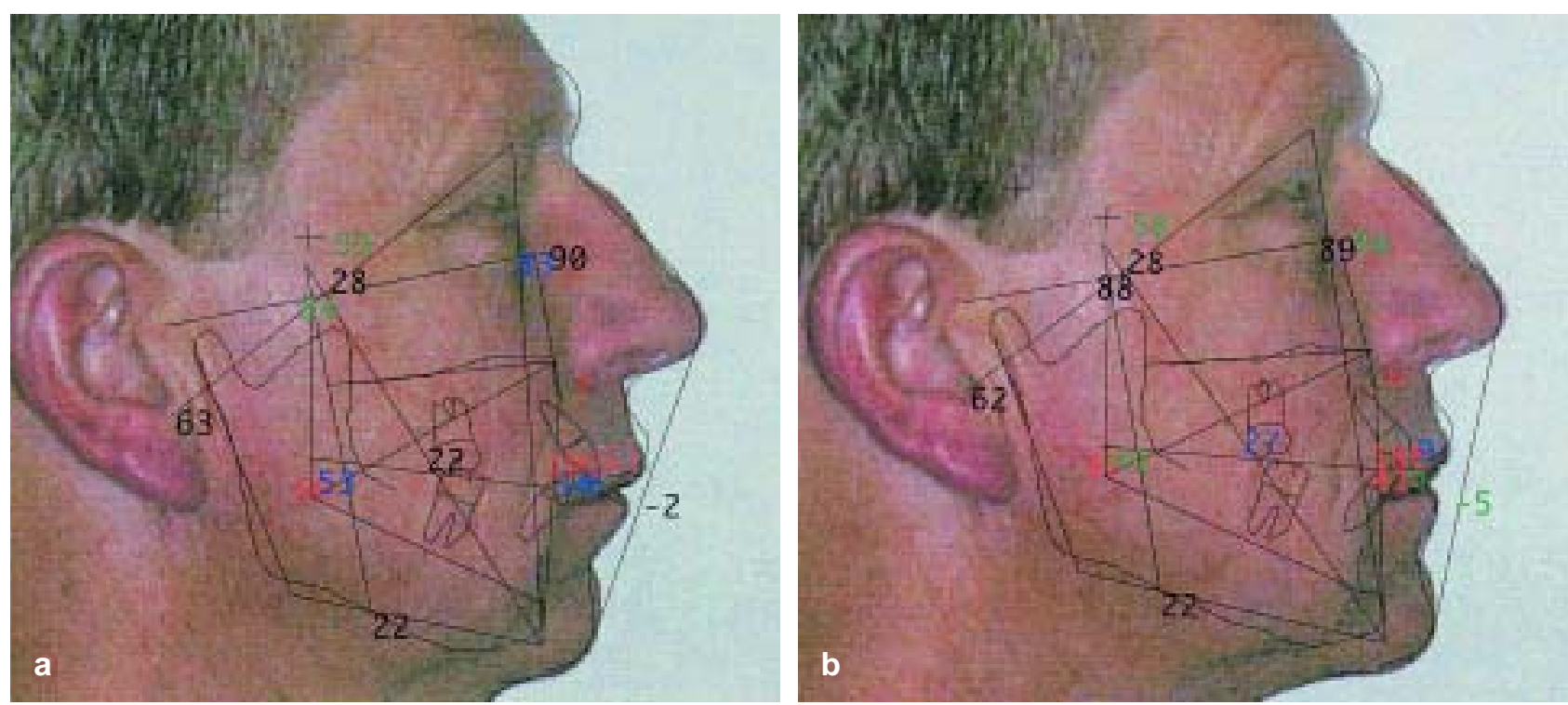

Fig 12. Computer simulation of a planned bimaxillary advancement: (a) pre-operative - note short mandible; and (b) post-operative predicted change in profile. 

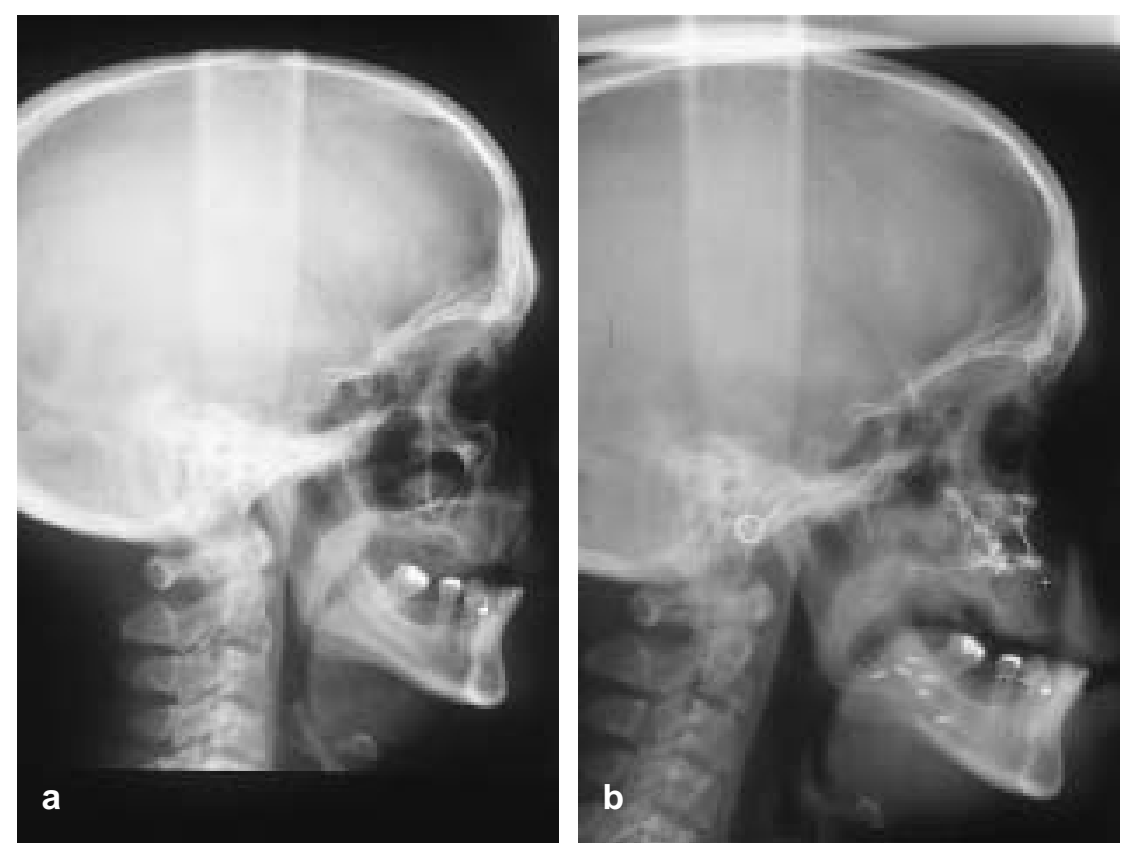

Fig 13. Lateral head cephalogram of a bimaxillary advancement: (a) pre-operative the patient is an achondroplastic dwarf and has marked midface retrusion; and (b) postoperative - the maxilla and mandible have been advanced by a Le Fort I and bilateral sagittal split osteotomies with internal fixation. Note the marked increase in the airway space.

improvement was noted, 67 per cent of patients had their RDI reduced to below $20 .^{40}$ The average advancement was $0.5 \mathrm{~cm}$ and there was no significant morbidity and no mortality.

In summary, treatment of OSA should be performed in a stepwise, multi-disciplinary fashion. Obvious functionality and social issues should be addressed along with pathology of the upper airway. The first treatment for OSA is nCPAP. If nCPAP fails, for whatever reason, then consideration can be given to MRA, particularly for patients with retrusive mandibles and tongue base obstruction. Obstruction at the palatal level can be addressed by UPPP but if the patient has a retrusive facial skeleton, then genioplasty or bimaxillary advancement should be considered. Good long-term results have been reported with bimaxillary advancement surgery.

At each stage, particularly if treatment is unsuccessful, multi-disciplinary re-evaluation and discussion are required. Patient involvement, with informed consent, is essential to success. There is no single guaranteed treatment and practitioners working in isolation not only may not succeed, they may cause their patients harm.

\section{ACKNOWLEDGEMENTS}

The authors wish to acknowledge the involvement of the staff and patients of the sleep units at the Royal Adelaide, Queen Elizabeth, Burnside and Daw Park Repatriation hospitals, in Adelaide, South Australia, in this study. The bimaxillary surgery illustrated in Figs 12 and 13 was performed by Dr RHB Jones, oral and maxillofacial surgeon, Royal Adelaide Hospital.

\section{REFERENCES}

1. Young T, Palta M, Dempsey J, et al. The occurrence of sleep disordered breathing amongst middle-aged adults. N Eng J Med 1993;328:1230-1235.

2. Oral devices for the reduction of snoring and sleep apnoea. Based on the guidelines of the American Sleep Disorders Association. Recommendations of the Australian Dental Association and the Thoracic Society of Australia and New Zealand. ADA News Bulletin. December 1997.

3. ADA pamphlet: Oral appliances therapy for snoring and obstructive sleep apnoea. Sydney: Mi-tec, 1999.

4. Pegram GV, Lucas E. Normal sleep and sleep disorders. Oral \& Maxillofacial Surgery Clinics of North America 1995;7:181-194.

5. Snyder F, Hobram JA, Morrison DF, et al. Changes in respiration, heart rate and systolic blood pressure in human sleep. J Appl Physiol 1964;19:417-422.

6. International classification of sleep disorders: diagnostic and coding manual. Diagnostic classification steering committee. Rochester: American Sleep Disorders Association 1990.

7. Guilleminault C, Tilkian A, Dement WC. The sleep apnoea syndromes. Ann Rev Med 1976;27:465-481.

8. Gould GA, White KF, Rhind GB, et al. The sleep hypopnoea syndrome. Am Rev Respir Dis 1988;137:895-898.

9. Guilleminault C, Connolly SV, Winle RE. Cardiac arrhythmia and conduction disturbances during sleep in 400 patients with sleep apnoea syndrome. Am J Cardiol 1983;52:490-494.

10. He J, Kryger MH, Zorrick JF, et al. Mortality and apnoea index in OSA. Experience in 385 male patients. Chest 1988;94:9-14.

11. Benaim P, Foucher A, Leroy M, et al. OSA syndrome in adults and cardiovascular risk. A review. Annales de Cardiologie et angeiologie 1992;41;531-539.

12. George CF, Smiley A. Sleep apnoea and automobile crashes. Sleep 1999;22:790-795.

13. Teran-Santos J, Jimenez-Gomez A., Cordero-Guevara J. Cooperative Group Burgos-Sandes. The association between sleep apnoea and the risk of traffic accidents. N Eng J Med 1999;340:872-851.

14. Findley L, Fabrizio M, Knight $H$, et al. Driving simulator performance in patients with sleep apnoea. Am Rev Respir Dis 1989;140:529-530. 
15. Aulrich M. Automobile accident in patients with sleep disorders. Sleep 1989;12:487-494.

16. Strohl KP. The biology of sleep apnoea. Sci and Med 1996;32-41.

17. Telakivi T, Kajaste S, Partien M, et al. Cognitive function in obstructive sleep apnoea. Sleep 1993;16:574-575.

18. Sleep PL, Gold AR, Myer DA. Weight loss in mild to moderately obese patients with obstructive sleep apnoea. Ann Intern Med 1985;105:850-861.

19. Browman CP, Sampson MG, Yolen SF, et al. Obstructive sleep apnoea and body weight. Chest 1984;85:435-436.

20. Mallampati SR, Gatt SP, Gagino LD, et al. Clinical sign to predict difficult intubation. Can Anaes Soc 1982;32:429-439.

21. Woodson BT, Wooten MR. Comparison of upper airway evaluations during wakefulness and sleep. Laryngoscope 1994;104:421-828.

22. Schwab RJ, Gupta KB, Gefter WB, et al. Upper airway and soft tissue anatomy in normal subjects and patients with sleep disordered breathing. Am J Respir Crit Care Med 1995;152:1673-1689.

23. Sherring D. Craniofacial morphology and obstructive sleep apnoea. MDS thesis, Adelaide Uni, 2001.

24. Croft CB, Pringle M. Sleep nasendoscopy; a technique of assessment in snoring and obstructive sleep apnoea. Clinical Otolaryngology 1991;16:504-509.

25. Cartwright R. Effect of sleep position on sleep apnoea severity. Sleep 1984;7:110-114.

26. Sullivan CE, Isra FG, Bertheon-Jones M. Reversal of OSA by continuous positive airway pressure applied through the nares. Lancet 1981;1:862-865.

27. Kribbs NB, Pack AJ, Kline LR, et al. Objective measurement of patterns of nasal CPAP use by patients with obstructive sleep apnoea. Am Rev Resp Dis 1993;147:887-895.

28. Sanders MH, Gruendl CA, Rogers RM. Patient compliance with nasal CPAP therapy for sleep apnoea. Chest 1986;90:330-333.

29. Cartwright RD, Samuelson C. The effects of non-surgical treatment for obstructive sleep apnoea. The tongue retaining device. JAMA 1982;248:705-709.

30. Thornton WK, Roberts DH. Non-surgical management of the obstructive sleep apnoea patient. J Oral Maxillofac Surg 1996;54:1103-1108.

31. Schmidt-Nowara W, Lowe A, Wiegard L, et al. Oral appliances for the treatment of snoring and obstructive sleep apnoea. A review. Sleep 1995;18:501-510.

32. An American Sleep Disorders Association report. Practice parameters for the treatment of snoring and obstructive sleep apnoea with oral appliances. Sleep 1995;18:511-513.

33. Chu G. A comparison between fixed and adjustable oral appliances for obstructive sleep apnoea. Research project poster, Flinders Medical School, 1999.

34. L'Estrange PR, Battagel JM, Harkness B, et al. A method of studying adaptive changes in the oropharynx to variation in mandibular position in patients with obstructive sleep apnoea. J Oral Rehab 1996;23:699-711.
35. Miyamoto K, Ozbek M, Lowe AA, et al. Mandibular posture during sleep in healthy adults. Arch Oral Biol 1998;43:269-275.

36. Miyamoto K, Ozbek M, et al. Vertical mandibular posture during sleep in patients with obstructive sleep apnoea. Arch Oral Biol 1999;44:657-664.

37. O'Sullivan RA, Hillman DR, Mateljan R, et al. Mandibular advancement splint. An appliance to treat snoring and obstructive sleep apnoea. Am J Resp Crit Care Med 1995;151:194-198.

38. Clark GT, John JW, Hong CN. Treating obstructive sleep apnea and snoring assessment of an anterior mandibular repositing device. J Am Dent Assoc 2000;131:765-771.

39. Fugita S. Pharyngeal surgery for obstructive sleep apnoea and snoring. Snoring and obstructive sleep apnoea. New York: Raven, 1987:101-128.

40. Riley R, Guilleminault C, Powell N, et al. Palatopharyngoplasty failure, cephalometric roentonograms and obstructive sleep apnoea. Otolaryngol Head \& Neck Surg 1985; 93:240-243.

41. Sher AE, Schectman KB, Picarillo JF. The efficacy of surgical modifications of the upper airway in adults with obstructive sleep apnoea. Sleep 1996;2:156-177.

42. Troell R J, Lik K, Powell N B, et al. Radiofrequency of the soft palate in snoring and sleep disordered breathing. Operative techniques in otolaryngology, head and neck surgery. 2000;11: 121-123.

43. Dimitroulis G. Obstructive sleep apnoea: the role of the oral and maxillofacial surgeon. Aust Dent J 1997;42:25-29.

44. Riley R, Powell N, Guilleminault C. Obstructive sleep apnoea and the hyoid: A revised surgical procedure. Otolaryngol Head \& Neck Surg 1994;111:717-721.

45. Riley R, Powell N, Guilleminault C. Inferior mandibular osteotomy and hyoid myotomy suspension. J Oral Maxillofac Surg 1989;47:159-164.

46. Yelegin O. Surgical management of obstructive sleep apnoea. A preliminary report. 14th convocation, RACDS. Auckland, NZ 2000.

47. Waite PD, Wooten V, Lachner J, et al. Maxillomandibular advancement surgery in 23 patients with sleep apnoea. J Oral Maxillofac Surg 1989;47:1256-1261.

48. Douglas NJ. Surgical treatment of obstructive sleep apnoea. Sleep Medicine Reviews 1997;1:77-86.

49. Riley R, Powell N, Kasey K, et al. Surgery and obstructive sleep apnoea: Long term clinical outcome. Otolaryngol Head \& Neck Surg 2000;122:415-421.

Address for correspondence/reprints: Professor Alastair Goss Oral \& Maxillofacial Surgery Unit Adelaide University Adelaide, South Australia 5005 